\title{
La formación de valores en los educandos
}

Patricia del Pilar Sánchez Cabezas Universidad Técnica de Babahoyo pattysanchez1984@hotmail.com

\section{Resumen}

El objetivo de este trabajo consistió en realizar una reflexión acerca de la formación de valores, su importancia, concepciones teóricas y vías que contribuyen a su realización. Para cumplir este objetivo se efectuó una revisión bibliográfica en la cual se consultaron trabajos realizados por distintos autores, entre ellos, tesis de maestría, doctorado y artículos publicados en revistas científicas de reconocido prestigio.

Los valores están vinculados a la moral, expresada como un sistema de normas, principios, ideales y cualidades, que denotan además los intereses y motivaciones de los individuos en la sociedad.

En este sentido, la escuela ocupa el lugar principal dentro del conjunto de influencias educativas que actúan en la formación de niños y jóvenes, pues el trabajo educativo se desarrolla en el aula y en los demás lugares donde actúan los alumnos.

Por tanto, la responsabilidad de la familia es de gran impor- tancia, ya que guía los primeros pasos del niño, quien va formando su personalidad con las relaciones que establece con su entorno y miembros de la comunidad.

Es conclusión, la existencia de un nuevo orden económico mundial significa un reto para enfrentar la formación de valores. Ante esta situación es urgente la acción educativa a través de todas las partes involucradas a fin de afrontar las contradicciones existentes y atenuar sus efectos.

Palabras clave: Educación en valores, formación, familia, escuela, comunidad.

\section{Abstract}

The aim of this study was to conduct a reflection on the formation of values, its importance, theoretical concepts and pathways that contribute to its realization. According to this objective the paper shows a literature review by different authors, including master and doctoral thesis and articles published in prestigious scientific journals.

Cómo citar este artículo: Sánchez P. (2015) La formación de valores en los educandos. CUMBRES, Revista Científica. 1(2) 38 - 43 
The values are linked to the moral, expressed as a system of rules, principles, ideals and qualities, which also denote interests and motivations of individuals in society.

In this sense, the school is the principal place within the set of educational influences, institution that acting on the education of children and youth, because the educational work is developed in the classroom and other places where students act.

Therefore, the responsibility of the family has a great importance, because it guides the first steps of the child, who is forming his personality in the relationship with his environment and community members.

In conclusion, the existence of a new world economic order means a challenge to the formation of values. In this situation is urgent the educational action by means of the work of all stakeholders in order to fight against the contradictions existed and mitigate its effects.

Keywords: Values education, training, family, school, community.

\section{Introducción}

Según (Rodríguez, 2011) el tema de los valores es una vieja preocupación del ser humano. A lo largo de la historia del pensamiento filosófico han sido diferentes posiciones teóricas (naturalismo, objetivismo, subjetivismo, sociologismo), las que han intentado dar una explicación a la naturaleza de los valores humanos. Sin embargo ninguna de las posiciones clásicas citadas logra brindar una teoría satisfactoria en cuanto al tema, pues cada caso asume una naturaleza distinta y única para los valores sin llegar a la esencia y complejidad de esta categoría.

Los problemas teóricos acerca de los valores son todavía el centro de agudas discusiones, pues aún desde una misma plataforma ideológica, filósofos y pensadores se contraponen en cuanto a la naturaleza y el papel de este fenómeno. De hecho, existen criterios en cuanto a la definición de valor, de su carácter y forma de manifestarse en el sujeto, lo que está en correspondencia con la particular concepción filosófica del mundo de los autores y la solución que ofrezca al primer aspecto del problema fundamental de la filosofía (Domínguez, 2011).

La filosofía marxista establece el análisis objetivo de los valores aplicados a la vida social de ahí que plantee que el valor existe en las formas valorativas de la conciencia como son la política, el arte, la moral y otras esferas de la actividad social y espiritual de los hombres, de forma espontánea en calidad de representaciones e imágenes o de objetos, fines y medios de la actividad práctico e histórico social (Rodríguez, 1986).

Muchas ciencias utilizan el término valor con un significado conceptual, estas son: la Biología, la Economía, la Estadística, la Lógica, la Pedagogía, la Psicología y la Sociología entre otras con multitud de acepciones y connotaciones específicas; de ahí el carácter polisemántico del término.

Toledo, (2011) hace un análisis de las principales causas que provocan la pérdida de algunos valores, que lo expresa de esta forma: "es un tema de gran actualidad en el mundo de hoy en el que ha aumentado la violencia, los asesinatos, las violaciones, la mayoría de la humanidad sin derecho a la educación y a la salud pública, el desempleo, la falta de atención social, la proliferación de las drogas, la prostitución, la destrucción del medio ambiente, los intereses de ganancias personales por encima de las necesidades sociales, la injusta distribución de las riquezas fomentándose la cultura del tener y no, del ser, lo que unido a la creciente globalización de las esferas sociales y sobre todo a la comunicación, se ve reflejado a escala internacional, la pérdida de la significación de los valores sociales".

Chacón, (1995) enfatiza en el proceso de formación de valores, el papel que tiene el educador, el colectivo pedagógico y el trabajo educativo y formativo de las nuevas generaciones, aborda la necesidad del tratamiento individual y colectivo de esta formación.

Martínez, (1999) aclara que de esta forma la formación y transmisión de valores se convierte en un aspecto imprescindible. En la actualidad se habla de Crisis de valores o pérdida de valores, estas proceden en momentos convulsos de rompimiento, ya sean positivos o negativos.

La crisis del socialismo y con ella la existencia de un mundo unipolar puso en crisis sin duda nuestro sistema de valores, trajo inseguridad, sentimiento de pérdidas de validez de aquello que se consideraba valioso y pudo cambiar de lugar la posición de los valores, se otorga mayor prioridad a valores negativos para nuestra sociedad, como el egoísmo, la deshonestidad, el individualismo, el consumismo incluso provocar la práctica de conductas distintas como la doble moral.

Otra de las problemáticas que presenta la formación de valores las señala (Baxter, 2007) cuando expresa que; la 
tendencia a separar la educación de la instrucción, a no aprovechar las potencialidades que brinda los contenidos como proceso en sí para incidir en la formación de sentimientos y valores, ha traído como consecuencia que el estudiante tenga muy pocas posibilidades de proyectarse en la clase, en las actividades curriculares; es decir; de participar de forma activa e independiente, de plantear sus puntos de vista, intereses, juicios, estados de ánimo y valoraciones acerca de todo hecho, fenómeno, objeto o proceso que tienen un significado, una repercusión social o un valor para ellos.

En resumen, todas estas reflexiones nos demuestran, en primer lugar el interés de los investigadores acerca de esta problemática, por su importancia para el desarrollo social, en segundo lugar tienen en común elevar la espiritualidad del individuo, la vinculan al conocimiento y hacen énfasis en la importancia de su formación en las nuevas generaciones, en tercer lugar se advierte de que constituye una necesidad para identificar y consolidar la nueva sociedad que construimos.

De acuerdo a este análisis general sobre la problemática analizada, se precisa como objetivo de este trabajo el de reflexionar sobre la importancia de la formación de valores, concepciones teóricas y vías que contribuyen a su realización.

Para cumplimentar este objetivo se efectuó una revisión bibliográfica, de trabajos realizados por distintos autores, entre ellos tesis de maestría, doctorado y artículos publicados en revistas científicas con prestigio reconocido, de forma tal que fue recopilada una información actual de la situación y problemas que presenta la formación de valores.

\section{Marco teórico}

Concepciones acerca del significado de los valores del ser humano. El tema de los valores ha sido tratado desde la filosofía juda cristiana a partir de la perspectiva ético normativa, y solo a finales del siglo XVIII y principios del siglo XIX el enfoque toma otro sentido. Etimológicamente la palabra "valor" proviene del verbo latino valere que significa disfrutar de buena salud, también significa ser valiente, hacer esfuerzo, ser fuerte. Los valores humanos participan de esta significación original de la palabra, puesto que ellos, en cuanto principio que orientan el comportamiento de la persona, exigen del individuo buena dosis de esfuerzo y valentía. (Llorens, 2006).

(Fabelo, 1989) define valor como "la significación positiva que se refleja en la conciencia social de los objetos, leyes, categorías y fenómenos de la realidad objetiva". Otros autores que trabajan la temática han conceptualizado los valores desde una posición más cercana a la pedagogía.

Baxter, (1999) conceptualiza al valor como "una compleja formación de la personalidad, contenida no solo en la estructura cognitiva, sino fundamentalmente en los profundos procesos de la vida social, cultural y en la concepción del mundo del hombre, que existe en la realidad, como parte de la conciencia social y en estrecha correspondencia y dependencia del tipo de sociedad en el que niños, adolescentes y jóvenes se forman".

Aclara que, desde el punto de vista pedagógico, esta formación debe lograrse como parte de la educación general, científica que reciben los adolescentes y jóvenes: como conocimiento, como producto del reconocimiento de su significación que se transforma en sentido personal y se manifiesta como conducta.

Rodríguez, (1995), señala que los valores son atributos conformados históricamente, que el ser humano le otorga a sus productos sociales en función de su utilidad. Estos están determinados en última instancia por un prisma ideológico cultural que el momento histórico social impone"

Los valores tienen una doble significación como fuerza motriz de la conducta: por una parte brindan una orientación para la toma de decisiones a nivel de toda la sociedad; por otra parte, son una orientación para la toma de decisiones personales o individuales (Rodríguez, 2011).

(Rodríguez, 2000) señala que, los valores no son estables y eternos, dependen en gran medida de las épocas históricas y sus tareas y de las etapas del desarrollo ontogenético, y que de esta forma los valores pueden ser descritos como:

- Principios o creencias

- Relativos o estados de existencia o a modelos de comportamiento

- Trascienden objetos, grupos de personas y situaciones específicas

- Orientan la elección o evaluación de comportamientos y acontecimientos

- Expresan intereses individuales o colectivos.

\section{La educación en valores}

Martínez, (1999) señala que en las condiciones actuales de lucha ideológica la formación de valores adquiere un pa-

\section{$40 \quad$ Cumbres}


pel determinante, ya que constituyen nexos mediadores entre la teoría y la práctica y muestran los niveles alcanzados o no por la conciencia social, y continua expresando que la educación en valores es un proceso sistemático, pluridimensional, intencional e integrado, y nos garantiza la formación y el desarrollo de la personalidad consiente; se concreta por medio de lo curricular extracurricular y en toda la vida universitaria.

En tal sentido (Baxter, 1989) explica que en la actualidad la educación en valores, es una problemática que preocupa y es objeto de análisis de políticos, especialistas y educadores de diferentes países, independientemente del régimen social existente; y es así, porque ello está indisolublemente vinculado a la propia existencia del ser humano, es decir al desarrollo de la personalidad, y a su condición de ser social.

Hoy día, los psicólogos, pedagogos, sociólogos y científicos sociales en general, abordan la temática de los valores, en medio de las grandes contradicciones que marcan la vida del hombre en el mundo actual, con lo que se revela su dimensión universal, consustancial al hombre y su cultura (Miranda, 1999).

Para (Toledo, 2011) la educación en valores es parte del proceso educativo en el que el contenido axiológico, de determinados hechos, formas de ser, manifestaciones de sentimientos, actuaciones o actitudes humanas, con una significación social buena, que convocan una relación de aprobación y reconocimiento (vigencia) en el contexto de las relaciones interpersonales, trasciende al nivel de la conciencia, forma parte del proceso de formación de la personalidad, toca de cerca un componente esencial del ser humano: la espiritualidad.

En tal sentido (Fabelo, 1995) plantea que los valores que se forman en la conciencia juvenil son el resultado de las influencias por un lado de los valores objetivos de la realidad social, con sus constantes dictados prácticos o de los institucionales que llegan al hombre en forma de discurso ideológico, político y pedagógico.

Chacón, (1995) plantea que en el proceso de formación de valores, el papel que tiene el educador, el colectivo pedagógico y el trabajo educativo y formativo de las nuevas generaciones, aborda la necesidad del tratamiento individual y colectivo de esta formación.

"La escuela ocupa el lugar principal dentro del conjunto de influencias educativas que actúan en la formación de niños y jóvenes, el trabajo educativo se desarrolla en el aula, en el laboratorio, en los talleres en el comedor, en los albergues y en las actividades políticas ideológicas, productivas, recreativas y culturales, es decir, la labor educativa dirige toda la actividad escolar. El corazón del Trabajo Educativo es la labor de los profesores, si esta funciona mal, funcionará mal todo el sistema de trabajo (Castro, 1987).

Corresponde a la universidad dar respuesta a las necesidades de la sociedad, en relación a la formación integral y profesional de las nuevas generaciones, desarrollar rasgos, cualidades y hábitos de conducta de este tipo de hombre, por ser la institución más preparada para la educación, formación y desarrollo de las nuevas generaciones (Toledo, 2011).

"Educar en valores es la acción planificada, orientada y controlada que ejercen las familias, la escuela y la comunidad, mediante el ejemplo personal en la actuación de todos los adultos responsabilizados con la tarea o encargo social de formar a las nuevas generaciones (Baxter, 2007).

\section{La formación de valores profesionales}

Para poder llegar a comprender qué son los valores profesionales es necesario comenzar por definir qué es profesión "La profesión es una actividad social cooperativa, cuya meta interna consiste en proporcionar a la sociedad un bien específico e indispensable para su supervivencia como sociedad humana, para lo cual se precisa el concurso de la comunidad de profesionales que como tales se identifican ante la sociedad" (Hirsch, 2002).

Al referirse al tema (Chacón, 2002) precisa que la moral profesional está, en indicar y orientar, bajo el prisma del sentido crítico de la realidad y autocrítico del individuo, qué es lo que hay que hacer en cada momento del desarrollo histórico de su actividad profesional en el contexto de la realidad social, ante cada dilema o conflicto u obligación como compromisos sociales contraídos.

(Villalón, 2005), (González, 2005) y (Longoria, 2005) apuntan que los valores profesionales deben ser formados desde el mismo estudio de la profesión argumentando lo siguiente: "los valores que se forman en la carrera, se consideran como el conjunto de valores que heredados de los más genuino de la creación universal, expresan, aglutinados por los valores profesionales, la significación práctica y el sentido de los resultados de la actividad material 
y espiritual de los estudiantes en el proceso de formación integral, de las carreras universitarias".

Rodríguez, (2011) explica que la formación de valores se produce mediante el vínculo de los componentes cognitivo, afectivo y conductual, sin desconocer el volitivo. El conocimiento como simple comprensión de la realidad cuando se convierte en reflexión personalizada incluye lo afectivo y por tanto contribuye a la formación del valor y a su vez las vivencias afectivas que el sujeto experimenta, contribuyen a formar el conocimiento.

A modo de conclusión de esta parte, se expone el análisis que hace (Ortiz, 1999) sobre algunos aspectos que, a su criterio, pueden afectar nuestro comportamiento en la sociedad: "En la actualidad vivimos en un mundo acelerado siempre pensando en la competitividad personal, profesional, comercial; en tener un nivel de estatus superior al otro, éste como una exigencia del mundo globalizado, sin embargo, no nos hemos detenido a pensar hasta donde nos puede llevar esa "superación personal" y continua expresando que, "Estar ocupados en la búsqueda de ser los mejores en todos los ámbitos en los cuales nos desenvolvemos, nos ha llevado al olvido de observar que esa transformación en sujetos individualistas aislados truncará nuestra acción como agentes sociales activos en nuestra vida cotidiana, lo anterior afecta diversos aspectos de nuestro entorno".

\section{Conclusiones}

1. Los valores están vinculados a la moral, expresada como un sistema de normas, principios, ideales y cualidades, expresan además los intereses y motivaciones de los individuos en la sociedad.

2. La escuela ocupa el lugar principal dentro del conjunto de influencias educativas que actúan en la formación de niños y jóvenes, pues el trabajo educativo se desarrolla en el aula y los demás lugares donde actúan los alumnos.

3. La responsabilidad de la familia es de gran importancia, ya que es quien guía los primeros pasos del niño, quien, con las relaciones que establece con su entorno y miembros de la comunidad, va formando su personalidad.

4. En la actualidad la formación de valores es objeto de análisis e investigación por parte de distintas discipli- nas de carácter social, entre las que se encuentran la filosofía, la Psicología, Pedagogía y la Sociología.

\section{Referencias bibliográficas}

- Álvarez, N y otros. (2000). La educación en valores del estudiante universitario a través del proceso docente educativo. Curso 8 Universidad (2000), La Habana, Cuba.

- Barrios, J. (1997). Democracia real versus democracia formal. Breve reflexión sobre la urgencia de una educación en valores. Universidad Complutense de Madrid.

- Baxter, E. (2007). Educar en valores, Tarea y reto de la sociedad. Editorial Pueblo y Educación. La Habana, Cuba

- Baxter, E. (1989). La formación de valores: Una tarea peᄀdagógica. La Habana: Ed. pueblo y educaᄀción, 40p.

- Cárdenas, N. (1999).

- Castro, F. (1987). Discurso pronunciado en la Clausura de la Graduación del V Contingente del Destacamento Pedagógico "Manuel Ascunce". Editorial Pueblo y Educación. La Habana.

- Chacón, N. (1995). "La formación de valores morales. Retos y perspectivas". Curso de superación IPLAE. MINED. Pedagogía 95. La Habana.

- Chacón, N. (2002). Dimensión Ética de la educación cubana., La Habana.

- Domínguez, W. (2005). La axiología martiana. Una vía para formar valores en la escuela primaria. Pedagogía (2005). Curso 97. ISBN 959-18-0107-6. Ciudad de La Habana, Cuba.

- Domínguez, W. (2011). La Filosofía de la Educación: un debate en torno a la formación de valores. Revista Electrónica Luz. Año X No. 3, (2011). ISSN 1814-151X. Holguín, Cuba.

- Fabelo, Y. (1995). "Intervención en la Audiencia pública sobre la formación de valores en las nuevas generaciones de la comisión y cultura, ciencia y tecnología de la Asamblea Nacional del Poder Popular". La Habana.

- Leal, H. (2000). Pensar, reflexionar y sentir en las clases de historia. Editorial Pueblo y Educación. La Habana Pág. 2.

- Llorens F. (2006). Ética y educación en los servicios de salud. Revista Cubana Salud Pública (2006); 32(4). Disponible en internet: http://bvs.sld.cu/revistas/spu/ vol32_4_06

- Martínez, B. (1999). El Proceso Educativo Integral, fuente inagotable, para la formación de valores. Experiencia Educativa en la carrera de Construcción del ISPETP. Tesis en opción al Título de Máster en Pedagogía Profesional. ISPETP. La Habana, Cuba.

\section{Cumbres}


- Miranda, T. (1999). Los niños y los valores. Una experiencia en la escuela. Instituto Pedagógico Latinoamericano y Caribeño. Congreso "Pedagogía 99".

- Ortiz, E. (1999). La Formación de Valores en la Educación Superior desde un Enfoque Psicopedagógico. Revista de Ciencias Pedagógicas, No. 20, Enero-Junio, La Habana.

- Pupo, R. (1989). “La actividad cono categoría filosófica”. Editorial Ciencias Sociales, La Habana.

- Rodríguez J, Oneida, G. Valdés y P. I. Rondón. (2011). Consideraciones teóricas sobre la Formación de Valores en los Estudiantes universitarios del municipio de Trinidad. Cuadernos de Educación y Desarrollo. Vol 3, No 29 (julio 2011), ISSN: 1989-4155. Universidad de Málaga. España.

- Rodríguez, C. (1995). La refundación universitaria de los valores humanos. Curso en Congreso "Pedagogía 95".

- Rodríguez, L. (1986). La ciencia y Valor. Editorial Pueblo y Educación. La Habana,

- Rodríguez, L. (1986). La ciencia y Valor. Editorial Pueblo y Educación. La Habana,

- Rodríguez, L. (1989).Filosofía, Ciencia y Valor. La Habana. Editorial Ciencias Sociales.

- Toledo, S. (2011). La educación en valores en el proceso educativo: concepción y sentido integral. Odiseo Revista Electrónica de Pedagogía, ISSN 1870-1477, Septiembre 8, (2011). Disponible en internet: www.odiseo.mx

- Villalón, My Barciela, M. González-Longoria. (2005). La formación de valores de los estudiantes universitarios. Curso "Pedagogía 05", La Habana, Cuba. 